\title{
El zooplancton de un lago somero hipereutrófico de la región central de Argentina: cambios después de una década
}

\author{
Santiago Andrés Echaniz, ${ }^{1,2}$, Alicia María Vignatti ${ }^{1} \&$ Pablo César Bunino ${ }^{1}$ \\ ${ }^{1}$ Departamento de Ciencias Naturales, Facultad de Ciencias Exactas y Naturales, \\ Universidad Nacional de La Pampa, \\ Av. Uruguay 151, 6300, Santa Rosa, Provincia de La Pampa, República Argentina, \\ e-mail:fexactas@unlpam.edu.ar,http://www.exactas.unlpam.edu.ar \\ ${ }^{2}$ Autor para correspondencia: Santiago Andrés Echaniz,e-mail: sechaniz@cpenet.com.ar
}

ECHANIZ, S.A., VIGNATTI, A.M. \& BUNINO, P.C. 2008. The zooplankton of a hipereutrophic shallow lake of the central region of Argentina: changes after one decade. Biota Neotrop. 8(4): http://www.biotaneotropica.org.br/v8n4/en/abstract?article+bn01008042008.

\begin{abstract}
The water bodies ecology is influenced by the contributions of nutrients from the basin. The shallow lentic ecosystems generally presents high levels of eutrophy, which allows to sustain a highly productive zooplanktonic fauna. According to the model of the alternative states of shallow lakes, zooplankton of these environments is characterized by a taxonomic composition, a spectrum of sizes and therefore a biomass that depends on the fishes present. Although in Argentina these ecosystems are abundant and of their importance given by their productivity, diversity and by their recreational and tourist interest, only recently they have begun to be studied. This contribution must by objective give information collected during 2006, by means of monthly samplings of water and zooplankton, on the factors of limnologic importance, their variation and influences on the water transparency and the abundance and zooplanktonic biomass of a shallow urban hipereutrophic lake of La Pampa province and to compare the situation registered with a similar study made between 1995 and 1996. The lagoon is characterized by low salinity and transparency and the reduction of these parameters between both periods studied. The nutrients concentration is greater than the verified in similar environments of Buenos Aires province. Although during both periods the species number was the same, it was verified changes in the taxonomic composition, registering in 2006 smaller number of cladocerans and greater of rotifers and the absence of Daphnia species, which can contribute to the reduction of the water transparency in this period. The found species were of small size, typical characteristic of zooplankton under fish predation. Between both periods a reduction in the abundance of the zooplankton community was verified, mainly between the cladocerans and rotifers.
\end{abstract}

Keywords: shallow lakes, zooplankton biomass, change, one decade after.

ECHANIZ, S.A., VIGNATTI, A.M. \& BUNINO, P.C. 2008. El zooplancton de un lago somero hipereutrófico de la región central de Argentina: cambios después de una década. Biota Neotrop. 8(4): http://www.biotaneotropica.org.br/v8n4/es/abstract?article+bn01008042008.

Resumen: La ecología de los cuerpos de agua está influenciada por los aportes de nutrientes desde la cuenca. Los ambientes lénticos de escasa profundidad generalmente presentan elevados niveles de eutrofia, lo que permite alojar una fauna zooplanctónica altamente productiva. Según el modelo de los estados alternativos de los lagos someros, el zooplancton de estos ambientes se caracteriza por una composición taxonómica, un espectro de tallas y por consiguiente una biomasa que dependen de la fauna íctica presente. A pesar de que en Argentina estos ambientes son abundantes y de su importancia dada por su productividad, diversidad e interés recreativo y turístico, sólo recientemente han comenzado a estudiarse considerando estas relaciones. Esta contribución tiene por objetivo dar información colectada durante 2006, mediante muestreos mensuales de agua y zooplancton, sobre los factores de importancia limnológica, su variación e influencia sobre la transparencia del agua y la abundancia y biomasa zooplanctónica de un lago somero urbano hipereutrófico de la provincia de La Pampa y comparar la situación registrada con un estudio similar realizado entre 1995 y 1996. La laguna se caracteriza por sus bajas salinidad y transparencia y por el descenso de estos parámetros entre los dos períodos estudiados. La concentración de nutrientes es mayor que la verificada en lagunas similares de la provincia de Buenos Aires. Si bien durante los dos períodos el número de especies fue el mismo, se verificaron cambios en la composición taxonómica, registrándose en 2006 menor número de cladóceros y mayor de rotíferos y la ausencia de especies de Daphnia, lo que puede contribuir al descenso de la transparencia verificada en este período. Las especies halladas fueron de talla pequeña, típico del zooplancton sometido a predación por peces. Entre ambos períodos se verificó un descenso en la abundancia de la comunidad zooplanctónica, sobre todo en cladóceros y rotíferos.

Palabras clave: lagos someros, biomasa zooplanctónica, cambio, una década después. 


\section{Introducción}

El estudio de los lagos someros fue encarado a principios de la década de 1990, generalmente en relación a procesos de eutrofización por el vertido de nutrientes en sus aguas por algunos autores como Jeppesen (1998), Jeppesen et al. (1991 y 2000), Scheffer (1998) y Scheffer et al. (1993), que con sus aportes han contribuido a conocer la importancia para los ecosistemas acuáticos, tanto de la entrada como de la carga interna de nutrientes y su influencia sobre la transparencia del agua y las productividades primaria y secundaria, al punto que llevó a plantear el modelo de los estados alternativos de los lagos someros.

Los lagos someros (lagunas) son cuerpos de agua generalmente ubicados en paisajes de llanura, que debido a su escasa profundidad no estratifican térmicamente, lo que les da un carácter polimíctico (Scheffer 1998). Generalmente tienen una elevada concentración de nutrientes (fósforo y nitrógeno), lo que ocasiona que sean ambientes eutróficos o hipereutróficos, con grandes biomasas en todos los niveles tróficos y tasas de producción primaria y secundaria también muy altas (Scheffer 1998). Además, en este tipo de ambientes los tiempos de permanencia del agua son muy variables, lo que ocasiona grandes cambios en la salinidad (Quirós et al. 2002a).

En Argentina, si bien existen estudios sobre aspectos ecológicos de los lagos someros, la mayoría se refieren a algún grupo taxonómico, funcional o nivel trófico en particular, como los trabajos de Ringuelet (1962, 1972), Ringuelet et al. (1967) en los que se describieron algunas lagunas de la pampasia bonaerense, haciendo referencia a las especies zooplanctónicas, y su relación con el contenido de sales del agua, los relevamientos que Olivier $(1955,1961)$ realizó en las lagunas Salada Grande y Vitel, también de la provincia de Buenos Aires, o los de José de Paggi (1976, 1980, 1983, 1984) y Paggi (1980), quienes estudiaron aspectos ecológicos de ambientes lénticos relacionados con la llanura de inundación del río Paraná, sujetos por lo tanto al régimen pulsátil del río.

Más recientes son las contribuciones de Claps et al. (2004) y de Quirós (2000), Quirós et al. (2002a, b, c) y Boveri \& Quirós (2002), quienes realizaron estudios en los que describieron la estructura y funcionamiento de cuerpos de agua someros situados en la provincia de Buenos Aires, sobre todo en la cuenca del río Salado, donde existen lagunas fuertemente influidas por la entrada de nutrientes aportados por el intenso uso de la tierra realizado en sus cuencas. Este último autor (Quirós 2000) realizó un relevamiento en el que mencionó la relación entre disponibilidad de nutrientes, clorofila y biomasa de zooplancton para algunos cuerpos de agua del país, pero tomó en cuenta los más conspicuos y de mayor interés pesquero. En la provincia de La Pampa sólo registró información de 2 cuerpos de agua (lagunas Urre Lauquen y La Dulce, ambas en el departamento Curacó), en muestreos puntuales realizados en el pico de la estación de crecimiento.

En la provincia de La Pampa, en la región central semiárida de Argentina, la ecología y la taxonomía de los lagos someros y de su zooplancton ha comenzado a estudiarse recientemente (Echaniz \& Vignatti 1996, 2001, 2002, Echaniz et al. 2005, 2006, Pilati 1997, 1999; Vignatti \& Echaniz 1999, Vignatti et al. 2007). Aunque en estos aportes se relacionaron las principales variables físico químicas, tales como temperatura, $\mathrm{pH}$, salinidad o contenido iónico, con la abundancia y composición taxonómica de la comunidad zooplanctónica, no se hizo referencia a la cantidad de nutrientes (fósforo y nitrógeno) presentes en el agua y su influencia sobre la biomasa del zooplancton.

La laguna Don Tomás es un cuerpo de agua hipereutrófico, ubicado en la ciudad de Santa Rosa. Está altamente afectada por la ciudad aledaña, dado que es el cuenco receptor del sistema de desagües pluviales. Reviste un interés particular, ya que por estar ubicada en un parque público tiene un importante uso recreativo, tanto por su interés paisajístico como porque en ella se desarrolla pesca deportiva.

Los objetivos de esta contribución son, por un lado, aportar información sobre la composición taxonómica del zooplancton, su variación y establecer relaciones entre la densidad y biomasa de los organismos con la concentración de clorofila $a$ y la disponibilidad de nutrientes a lo largo del año 2006 en la laguna Don Tomás y por otro lado, dado que se cuenta con información colectada en estudios anteriores, realizados entre marzo de 1995 y febrero de 1996 (Echaniz \& Vignatti 2001), se comparó el estado actual de la laguna con el registrado hace 10 años.

\section{Material y Métodos}

Se realizaron muestreos mensuales, durante el período comprendido entre febrero de 2006 y diciembre de 2006. En cada uno de ellos se tomaron dos muestras cuantitativas de zooplancton empleando una trampa de Schindler-Patalas de 10 litros de capacidad, provista de una red de $0,04 \mathrm{~mm}$ de abertura de malla y una muestra cualitativa, con una red de $22 \mathrm{~cm}$ de diámetro de boca y $0,04 \mathrm{~mm}$ de abertura de malla. Todas las muestras se anestesiaron con $\mathrm{CO}_{2}$ previo a la fijación, a los efectos de evitar contracciones que deformen los ejemplares y distorsionen las medidas necesarias para el cálculo de la biomasa (José de Paggi \& Paggi 1995).

En cada estación se determinaron la temperatura del agua y la concentración de oxígeno disuelto, mediante un oxímetro digital Lutron OD 5510 y la transparencia del agua mediante un disco de Secchi de $22 \mathrm{~cm}$ de diámetro y se tomaron muestras de agua para la determinación del $\mathrm{pH}$ mediante un pehachímetro digital Cornning PS 15, conductividad con un conductímetro Oakton TDSTestr 20 y salinidad mediante el método de residuo sólido. La concentración de clorofila $a$ se determinó por el método espectrofotométrico (Arar 1997; APHA 1999), la de nitrógeno total mediante el método de Kjeldahl y la de fósforo total mediante la digestión de la muestra con persulfato de potasio en medio ácido y espectrofotometría (APHA 1999).

A efectos de conocer la composición iónica, se efectuó un análisis químico en un laboratorio comercial del medio. Además, se determinó el contenido de sólidos suspendidos totales (seston), en sus diferentes fracciones (total, orgánico e inorgánico), mediante el filtrado de un volumen de agua conocido, a través de filtros tipo Whatman CFC, secados en estufa a $103-105^{\circ} \mathrm{C}$ hasta peso constante y posteriormente calcinados a $550{ }^{\circ} \mathrm{C}$ (EPA 1993).

Mediante el empleo de un microscopio óptico, dotado de un ocular micrométrico se tomaron las medidas convencionales de ejemplares de cada especie presente con un aumento constante de 100x.

La determinación taxonómica de los copépodos se efectuó siguiendo los criterios de Ringuelet (1958), Bayly (1992a, b), Reid (1985) y Gómez et al. (2004), los cladóceros según Goulden (1968), Smirnov (1971), Korovchinsky (1992) y Paggi (1995, 1998) y las determinaciones de los rotíferos según Ruttner-Kolisko (1974), Koste (1978) y Segers (1995).

Los recuentos de macrozooplancton se realizaron bajo microscopio estereoscópico a 20 y 40x y los de microzooplancton con microscopio óptico convencional, con 40 y 100x, en cámaras de Bogorov y Sedgwick-Rafter respectivamente. Las alícuotas se tomaron empleando submuestreadores de Russell de $5 \mathrm{~mL}$ y micropipetas de $1 \mathrm{~mL}$ y los recuentos fueron facilitados por la tinción con rosa de Bengala (José de Paggi \& Paggi 1995). Para determinar la biomasa del zooplancton se emplearon fórmulas que relacionan la longitud total con el peso seco de los ejemplares (José de Paggi \& Paggi 1995, Ruttner-Kolisko 
1977, Dumont et al. 1975, Rosen 1981, McCauley 1984, Culver et al. 1985, Kobayashi 1997 y Pilati \& Martínez 2003).

Se calcularon los índices de estado trófico en base a la transparencia del agua y concentraciones de clorofila y fósforo total (Carlson \& Simpson 1996).

Mediante el empleo de fotografías satelitales se determinaron los principales parámetros morfométricos, largo y ancho máximos, longitud de la línea de costa (Dangavs 1995) y mediante sondeos, la profundidad máxima de la laguna.

Se calcularon coeficientes de correlación de Pearson y se realizaron test de Levene a efectos de verificar igualdad de varianzas, análisis de varianza (ANOVA) y test de Kruskal Wallis en los casos correspondientes (Zar 1996).

\section{Resultados}

\section{Descripción del área de estudio}

La laguna Don Tomás (64 19’ 03” W y 36³7’20” S) es un cuerpo de agua somero, situado al oeste de la ciudad de Santa Rosa (Figura 1), de la que recibe el aporte de agua de los desagües pluviales, lo que permitió, que si bien en el pasado fuera un ambiente temporario, en la actualidad sea permanente. A efectos de evitar inundaciones en las zonas bajas de la ciudad, posee un sistema de bombas, que se pone en funcionamiento cuando existe riesgo de desbordes y envía el agua excedente a un cuenco situado al sur de la ciudad.

En sus alrededores se sitúa un Parque Recreativo de uso comunitario y en ella se desarrolla una fauna íctica integrada por Odonthestes bonariensis (Cuvier y Valenciennes, 1835) (pejerreyes), Cyprinus carpio (Linnaeus, 1758) (carpas), Cnesterodon decemaculatus (Jenyns, 1842) (mojarra), Jenynsia lineata (Jenyns, 1842) (mojarra) y Oligosarcus jenynsi (Günther,1864) (dientudos), lo que le confiere un alto valor turístico y recreativo.

Es una laguna que tiene una profundidad máxima de 2,3 m y una superficie de 135,2 ha. Su largo y ancho máximos son 1565 y $1181 \mathrm{~m}$. La longitud de la línea de costa es de $4725 \mathrm{~m}$ y el cálculo del desarrollo de la línea de costa $(1,14)$ muestra que su contorno es muy regular, casi circular y sin accidentes.

Dentro del cuenco principal en donde se realizó la toma de las muestras, no se registró vegetación arraigada. El fitoplancton de la laguna Don Tomás se caracteriza por su elevada diversidad, estando dominado por las clorofitas y cianofitas, 47,8 y $32,7 \%$ del total de las especies registradas respectivamente, entre las que se destacan

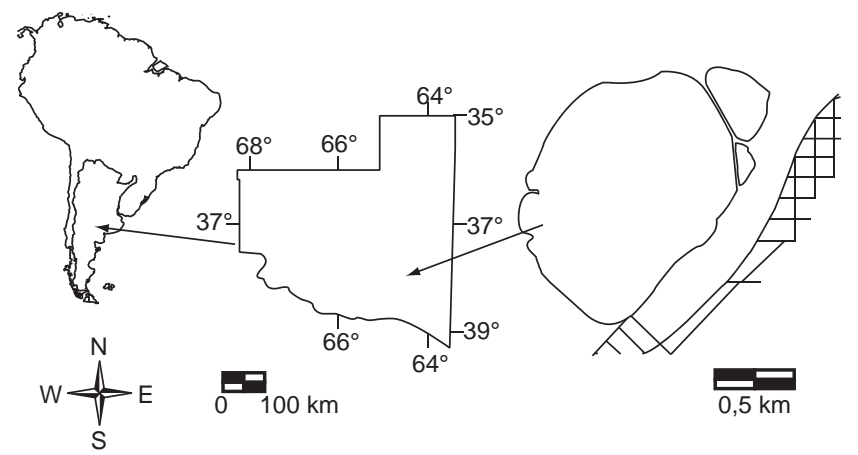

Figura 1. Croquis de la laguna Don Tomás, mostrando su ubicación geográfica en la provincia de La Pampa, en la región central de Argentina.

Figure 1. Sketch of Don Tomás pond, showing their geographical ubication in La Pampa province, central Argentina.
Anabaenopsis arnoldii Aptekarj, Plankthotrix agardhii (Gom.) Anagh. et Kom., Chlorella elipsoidea Gern. y Phormidium sp. entre otras (Alvarez et al. 2006).

\section{Parámetros ambientales}

La temperatura del agua varió en un amplio rango en función de la época del año (Figura 2). La máxima se registró durante noviembre y diciembre $\left(24,1^{\circ} \mathrm{C}\right)$ y la mínima en julio $\left(7,1^{\circ} \mathrm{C}\right)$. La concentración de oxígeno disuelto no presentó deficiencias y durante el periodo de estudio los valores fluctuaron entre 7,4 (marzo) y 10,3 (mayo) (Figura 2).

La transparencia del agua fue siempre reducida (Figura 3), oscilando entre $0,12 \mathrm{~m}$ en marzo y noviembre y $0,2 \mathrm{~m}$ en octubre, con una media a lo largo del período de estudio de $0,15 \mathrm{~m}$. Teniendo en cuenta que la profundidad promedio de la laguna fue de $1,15 \mathrm{~m}$, el cálculo de la relación $\mathrm{Z}_{\text {promedio }} / \mathrm{Z}_{\text {fótico }}$ (Quirós 2002) arrojó el valor de 2,35.

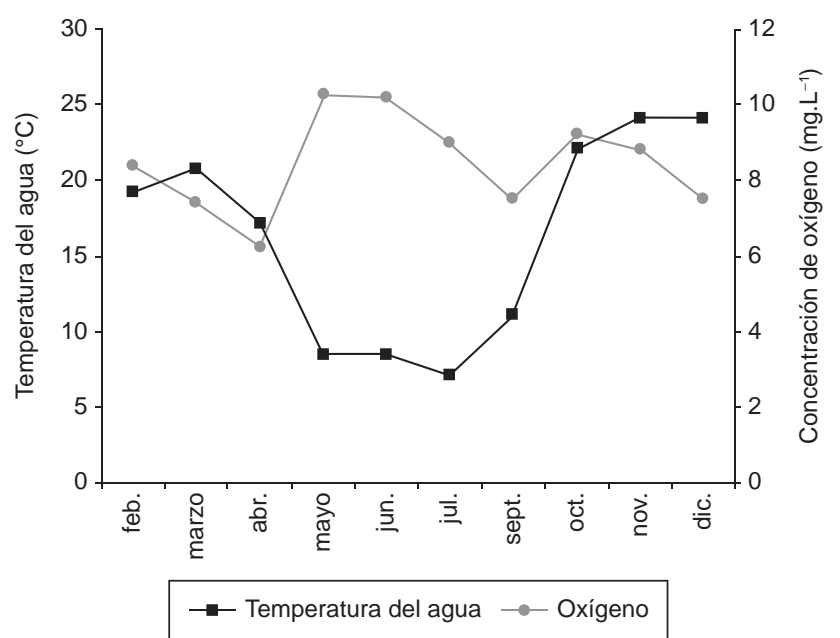

Figura 2. Variación de la temperatura del agua $\left({ }^{\circ} \mathrm{C}\right)$ y de la concentración de oxígeno disuelto (mg. $\left.\mathrm{L}^{-1}\right)$ en la laguna Don Tomás, entre febrero y diciembre de 2006.

Figure 2. Variation in water temperature $\left({ }^{\circ} \mathrm{C}\right)$ and dissolved oxygen concentration $\left(\mathrm{mg} . \mathrm{L}^{-1}\right)$ in Don Tomás pond, between February and December 2006.

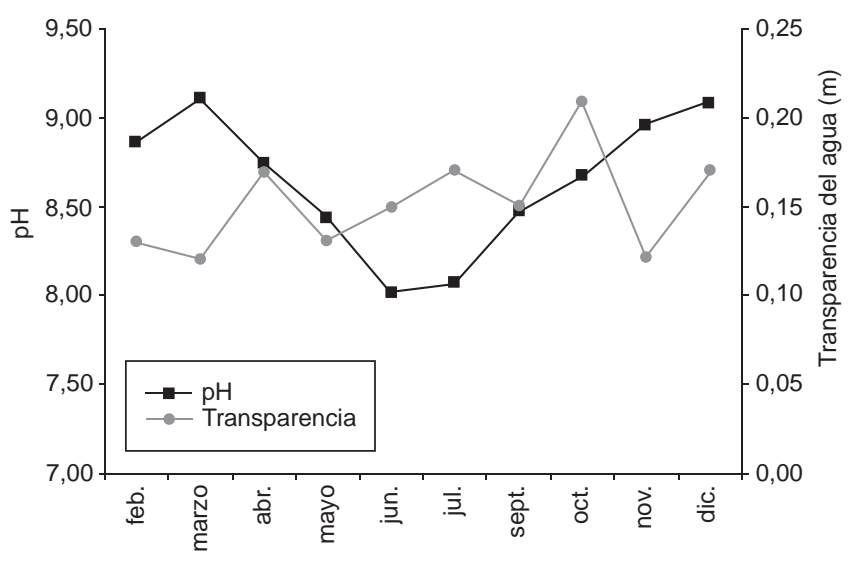

Figura 3. Variación del pH y la transparencia del agua (m) en la laguna Don Tomás, durante el período febrero a diciembre de 2006.

Figure 3. Variation in $\mathrm{pH}$ and transparency of the water (m) in Don Tomás pond, during the period February to December 2006. 
El contenido de sólidos suspendidos totales (seston total) determinado (Figura 4), mostró un valor medio de 54,58 mg.L $\mathrm{L}^{-1}$, siendo 51,29 y $3,28 \mathrm{mg} . \mathrm{L}^{-1}$ los valores medios de las fracciones orgánica (Figura 4) e inorgánica respectivamente.

La salinidad registrada durante el período de estudio fue baja y relativamente constante, variando entre 0,71 g.L $\mathrm{L}^{-1}$ (febrero) y $0,96 \mathrm{~g} . \mathrm{L}^{-1}$ (noviembre). Durante el estudio efectuado entre marzo de 1995 y febrero de 1996 (Echaniz \& Vignatti 2001) la salinidad media registrada fue el doble de la medida durante 2006 , con 1,65 g.L - $^{-1}$ contra 0,8 g.L. $\mathrm{L}^{-1}$ respectivamente. Luego de realizado el test de Levene $(p=0,074677$; $\mathrm{F}=3,536455)$, el análisis de varianza mostró que la diferencia entre ambos períodos es significativa ( $\mathrm{p}=0,000000 ; \mathrm{F}=307,383$ ). $\mathrm{El} \mathrm{pH}$, aunque siempre fue relativamente elevado mostró oscilaciones, ya que varió entre 8,01 (junio) y 9,11 (marzo) (Figura 3).

La concentración de clorofila $a$ determinada a lo largo del período de estudio fue elevada y registró una media de $154,6 \mathrm{mg} . \mathrm{m}^{-3}$. Fue mínima durante los meses de invierno, cuando alcanzó 88,78 mg.m ${ }^{-3}$ (julio) y máxima en verano cuando llegó a 211,46 mg.m $\mathrm{m}^{-3}$ (diciembre) (Figura 4).

El análisis del contenido iónico realizado en una muestra de agua tomada en noviembre (Figura 5) mostró que Don Tomás es una laguna bicarbonatada sódica. El cálculo de la relación entre las concentraciones de cationes mono y bivalentes $\left(\mathrm{Na}^{+}+\mathrm{K}^{+} / \mathrm{Ca}^{++}+\mathrm{Mg}^{++}\right)$ (Golterman 1978) indicó el predominio de los cationes monovalentes, ya que arrojó un valor de 3,86 .

Las concentraciones de nutrientes registradas durante el período estudiado, tanto la de fósforo como de nitrógeno fueron altas, oscilando entre 3,75 y $16,9 \mathrm{mg} . \mathrm{L}^{-1}$ para el fósforo (media 9,71 mg. $\mathrm{L}^{-1}$ ) y 7,5 y 16,88 mg.L $\mathrm{L}^{-1}$ para el nitrógeno (media 11,52 mg.L $\mathrm{L}^{-1}$ ) (Figura 6).

Los valores calculados de los índices de estado trófico que toman en cuenta la transparencia del agua, concentración de clorofila $a$ y fósforo total (Carlson \& Simpson 1996) fueron 87,3; 80,1 y 136,5 respectivamente.

\section{Zooplancton}

Se hallaron 4 especies de cladóceros, 4 de copépodos y 12 de rotíferos (Tabla 1). Entre los cladóceros, Bosmina huaronensis Delachaux, 1918 fue la única especie presente durante todo el período

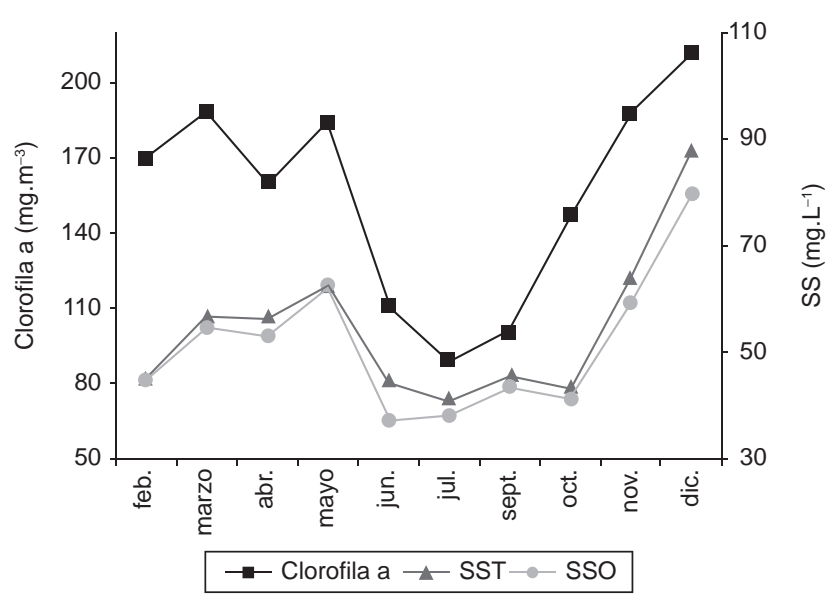

Figura 4. Variación de la concentración de clorofila $a\left(\mathrm{mg}^{-3} \mathrm{~m}^{-3}\right)$ y del contenido de sólidos suspendidos totales y de origen orgánico $\left(\mathrm{mg} . \mathrm{L}^{-1}\right)$, en la laguna Don Tomás, entre febrero y diciembre de 2006.

Figure 4. Variation in the concentration of chlorophyll $a\left(\mathrm{mg} \cdot \mathrm{m}^{-3}\right)$ and the content of suspended solids, totals and organics $\left(\mathrm{mg}^{\mathrm{L}} \mathrm{L}^{-1}\right)$ in Don Tomás pond, between February and December 2006. de estudio, siendo además dominante numéricamente. Esta especie registró su abundancia máxima en noviembre, con 346,67 ind. $\mathrm{L}^{-1}$ y mínima en mayo con 0,66 ind. $\mathrm{L}^{-1}$. La biomasa máxima fue de $2295,55 \mu \mathrm{g} . \mathrm{L}^{-1}$ (Figura 7 ) y la mínima de $0,35 \mu \mathrm{g}$.L $\mathrm{L}^{-1}$ cuando representaron el 95,3 y $24 \%$ del total de la biomasa de la taxocenosis de cladóceros respectivamente (Figura 8). Moina micrura Kurz, 1874, segunda en importancia, no se registró en los meses de junio, julio, septiembre y octubre. Su abundancia máxima se registró en febrero, con 75 ind. $\mathrm{L}^{-1}$ y la biomasa máxima en diciembre, con $452,12 \mu \mathrm{g} . \mathrm{L}^{-1}$ (Figura 7), cuando representó el $70 \%$ del total de la taxocenosis (Figura 8). Diaphanosoma birgei Korínek, 1981 y Alona sp. se registraron sólo en los meses de verano (Figura 7), alcanzando la primera una abundancia y biomasa máximas de 55 ind.L $\mathrm{L}^{-1}$ y $129,95 \mu \mathrm{g} . \mathrm{L}^{-1}$ en enero y la segunda 28,33 ind. $\mathrm{L}^{-1}$ y $34,3 \mu \mathrm{g} . \mathrm{L}^{-1}$ en noviembre (Figura 7). En el caso de D. birgei la biomasa del mes de febrero representó el 38,9\% del total de la taxocenosis (Figura 8).

Dentro de los copépodos el calanoideo Boeckella gracilis Daday, 1902 y el harpacticoideo Cletocamptus deitersi (Richard, 1897) se

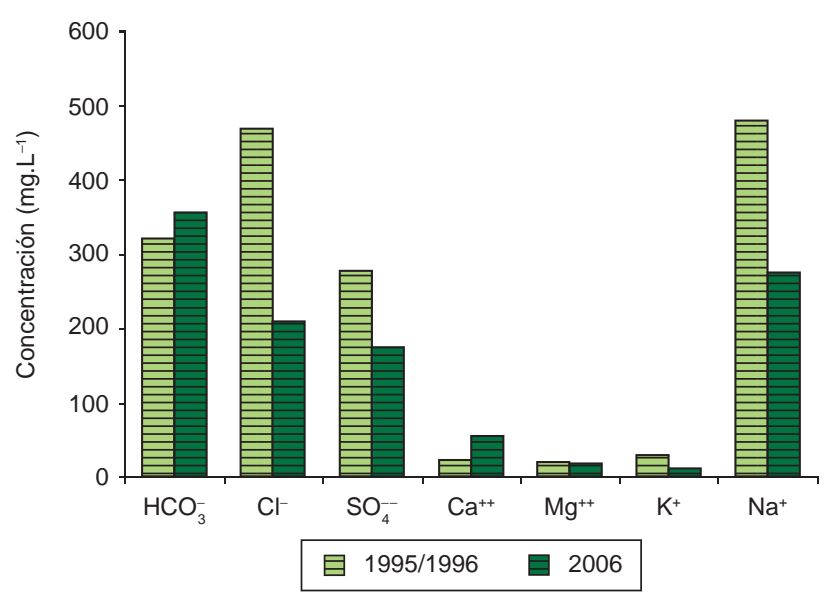

Figura 5. Comparación del composición iónica del agua de la laguna Don Tomás, registrados durante 1995-96 y 2006.

Figure 5. Comparison of the ionic composition of the water of Don Tomás pond, recorded during 1995-96 and 2006

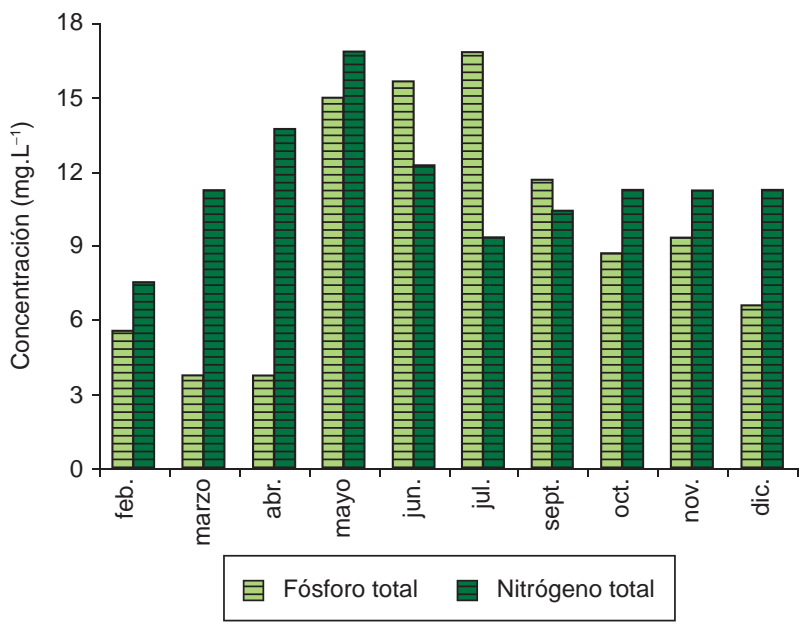

Figura 6. Variación de la concentración de fósforo y nitrógeno totales (mg.L $\mathrm{L}^{-1}$ ) en el agua de la laguna Don Tomás, durante el período febrero a diciembre de 2006.

Figure 6. Variation in the concentration of total nitrogen and phosphorus $\left(\mathrm{mg} . \mathrm{L}^{-1}\right)$ in the water of Don Tomás pond, during the period FebruaryDecember 2006. 
Tabla 1. especies registradas durante 2006 y frecuencia de aparición en las muestras (\%).

Table 1. registered species during 2006 and frequency of appearance in the samples $(\%)$.

\begin{tabular}{|c|c|c|}
\hline Especie & 1995/96 & 2006 \\
\hline \multicolumn{3}{|l|}{ Rotíferos } \\
\hline Keratella sp. & 92 & 100 \\
\hline Brachionus plicatilis Müller, 1786 & 75 & 70 \\
\hline B. dimidiatus (Schmarda, 1854) & - & 70 \\
\hline B. quadridentatus Hermann, 1783 & - & 30 \\
\hline B.angularis Gosse, 1851 & 25 & 30 \\
\hline B. havanaensis Rousselet, 1911 & 58 & 20 \\
\hline B. pterodinoides (Rousselet, 1913) & - & 20 \\
\hline B. caudatus Barrois \& Daday 1894 & 100 & 20 \\
\hline B. calicyflorus (Pallas, 1766) & - & 10 \\
\hline Hexarthra sp. & - & 30 \\
\hline Polyarthra sp. & 58 & 40 \\
\hline Filinia longiseta (Ehrenberg 1834) & 42 & - \\
\hline Colurella sp. & 67 & - \\
\hline Lecane sp. & - & 40 \\
\hline \multicolumn{3}{|l|}{ Cladóceros } \\
\hline Bosmina huaronensis Delachaux, 1918 & 100 & 100 \\
\hline Moina micrura Kurz, 1874 & 58 & 70 \\
\hline Diaphanosoma birgei Korínek, 1981 & 8 & 30 \\
\hline Alona sp. & 50 & 50 \\
\hline Daphnia spinulata Birabén, 1917 & 33 & - \\
\hline D. obtusa Kurz, 1874 & 17 & - \\
\hline Ceriodaphnia dubia Richard, 1895 & 33 & - \\
\hline Leydigia leydigi Schoedler, 1863 & 33 & - \\
\hline Macrothrix sp. & 33 & - \\
\hline \multicolumn{3}{|l|}{ Copépodos } \\
\hline Metacyclops mendocinus Wierzejski 1892 & 100 & 100 \\
\hline Microcyclops anceps Richard 1897 & 100 & 100 \\
\hline Boeckella gracilis Daday, 1902 & - & 20 \\
\hline Cletocamptus deitersi (Richard, 1897) & 8 & 10 \\
\hline
\end{tabular}

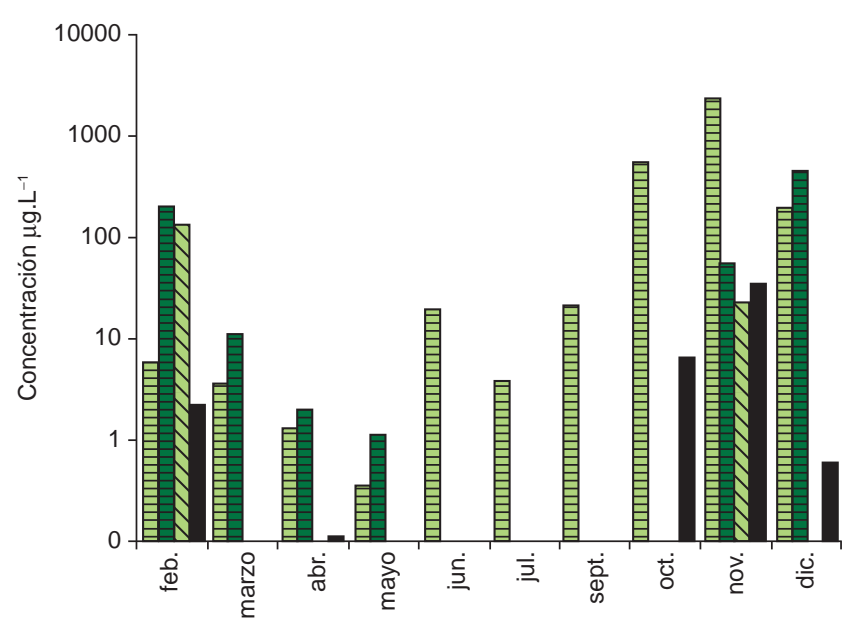

目B. huaronensis 目 M. micrura $\triangle D$. birgei Alona sp.

Figura 7. Variación de la biomasa $\left(\mu \mathrm{g} . \mathrm{L}^{-1}\right)$ de las especies de cladóceros dominantes en la laguna Don Tomás, entre febrero y diciembre de 2006.

Figure 7. Variation of biomass $\left(\mu \mathrm{g} . \mathrm{L}^{-1}\right)$ of the cladocerans dominant species in Don Tomás pond, between February and December 2006. colectaron sólo en septiembre, cuando presentaron abundancias y biomasas muy reducidas, 1,66 ind. $\mathrm{L}^{-1}$ y $100,46 \mu \mathrm{g} . \mathrm{L}^{-1}$ en el caso de B. gracilis y 1,05 ind. $\mathrm{L}^{-1}$ y $4,41 \mu \mathrm{g} . \mathrm{L}^{-1}$ para $C$. deitersi. En cambio, los ciclopoideos y las larvas nauplio se registraron en todo el período de muestreo. La abundancia máxima de adultos y copepoditos fue de 1066,67 ind. $\mathrm{L}^{-1}$ en marzo y la mínima de 92,43 ind. $\mathrm{L}^{-1}$ en mayo. Respecto a su biomasa, fluctuó entre 4108,19 y 365,96 $\mu \mathrm{g} . \mathrm{L}^{-1}$ en septiembre y febrero respectivamente (Figura 9). Los nauplios tuvieron una abundancia mínima de 18,33 ind.L $\mathrm{L}^{-1}$ en mayo y máxima de 1505 ind. $\mathrm{L}^{-1}$ en febrero, mientras que la biomasa varió entre 748,71 y $3,17 \mu \mathrm{g} . \mathrm{L}^{-1}$ en diciembre y mayo respectivamente (Figura 9).

Respecto a los rotíferos, Keratella sp. fue la única presente en todo el período de estudio y la más importante numéricamente. Su abundancia presentó un máximo de 1196,67 ind.L . $^{-1}$ en noviembre y

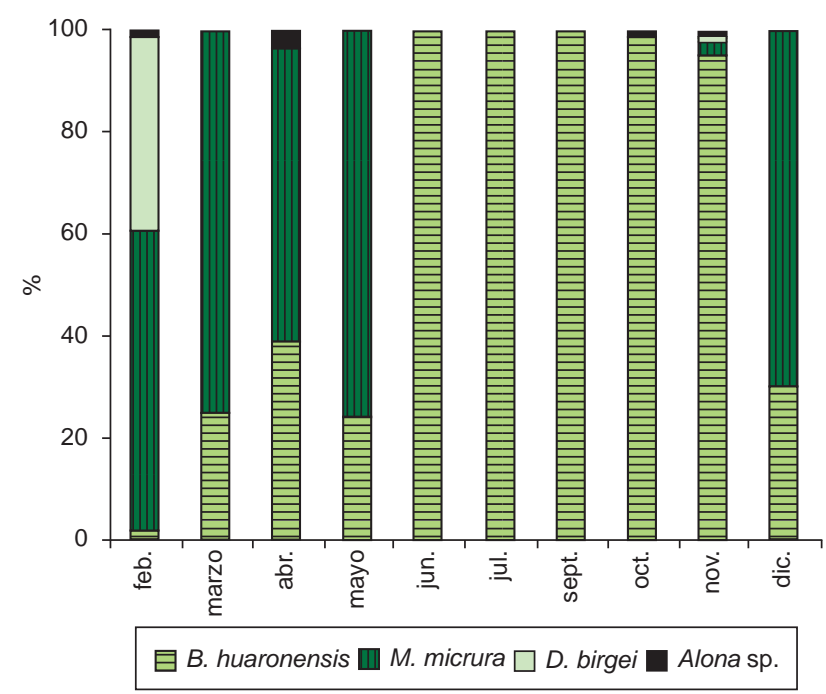

Figura 8. Aporte porcentual de biomasa $\left(\mu \mathrm{g} . \mathrm{L}^{-1}\right)$ de las especies de cladóceros dominantes en la laguna Don Tomás, entre febrero y diciembre de 2006.

Figure 8. Percentage contribution of biomass $\left(\mu \mathrm{g} . \mathrm{L}^{-1}\right)$ of the cladocerans dominant species in Don Tomás pond, between February and December 2006.

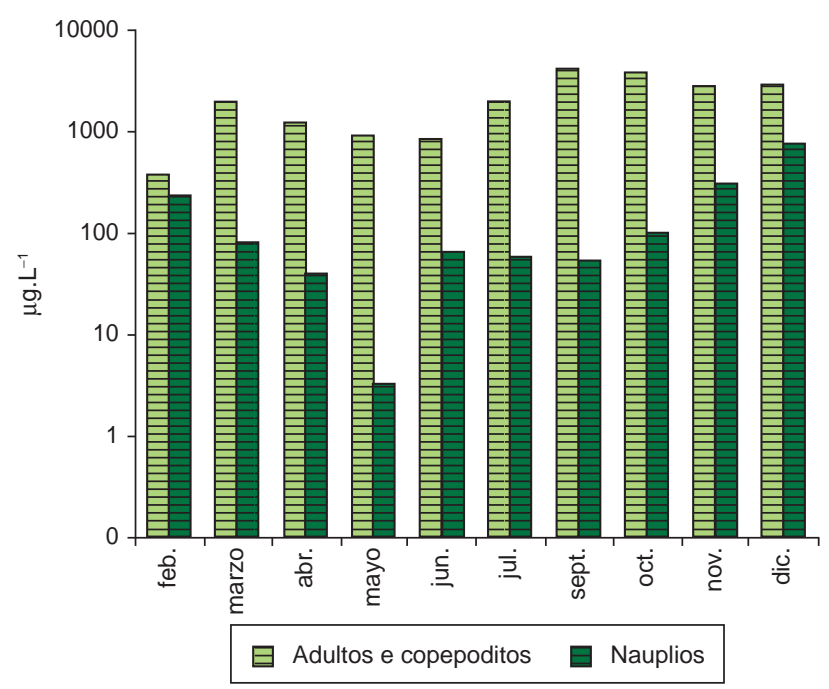

Figura 9. Variación de la biomasa $\left(\mu \mathrm{g} . \mathrm{L}^{-1}\right)$ de los copépodos ciclopoideos en la laguna Don Tomás, entre febrero y diciembre de 2006.

Figure 9. Variation of biomass $\left(\mu \mathrm{g} . \mathrm{L}^{-1}\right)$ of the ciclopoids copepods in Don Tomás pond, between February and December 2006. 
un mínimo de 1,67 ind. $\mathrm{L}^{-1}$ en mayo mientras que su biomasa fue de $624,47 \mu \mathrm{g} . \mathrm{L}^{-1}$ en noviembre y de $0,71 \mu \mathrm{g} . \mathrm{L}^{-1}$ en mayo (Figura 10). Brachionus plicatilis Müller, 1786, la especie más importante en términos de biomasa después de Keratella sp. no estuvo presente en junio, septiembre y octubre. Su abundancia y biomasa máximas (Figura 10) se registraron en abril, 1660 ind. $\mathrm{L}^{-1}$ y $360,31 \mu \mathrm{g} . \mathrm{L}^{-1}$ y las mínimas en mayo con 1,67 ind. $\mathrm{L}^{-1}$ y $1,96 \mu \mathrm{g} . \mathrm{L}^{-1}$. El resto de las especies se registraron a fines de primavera y verano, alcanzando densidades y biomasas reducidas en comparación con Keratella sp. y B. plicatilis.

Considerando la comunidad completa, el mayor aporte de biomasa fue hecho por los copépodos, $78,1 \%$ del total, seguidos por los cladóceros con $13,8 \%$. El aporte de los rotíferos fue reducido, ya que representó el 8,1\% del total (Figura 11).

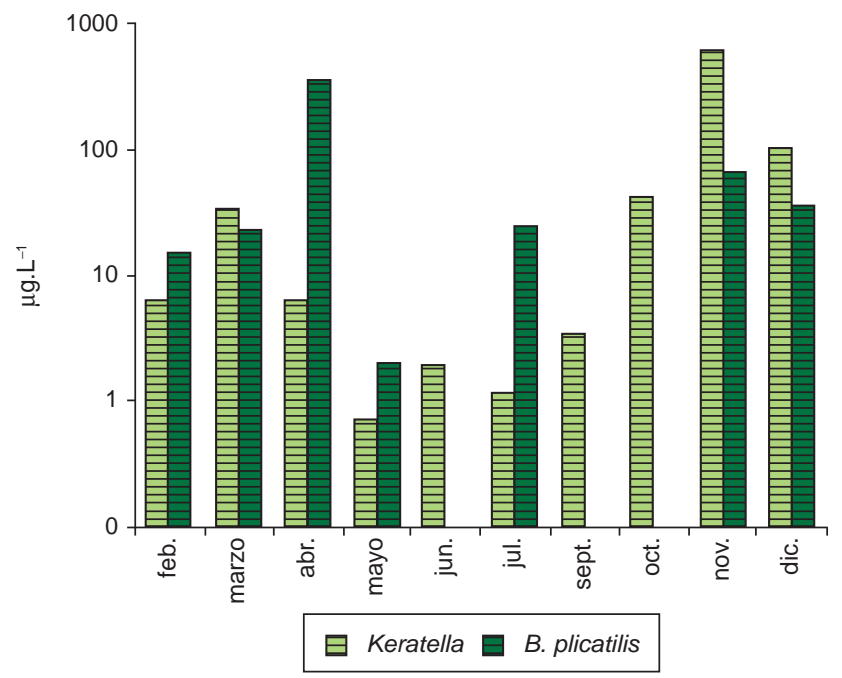

Figura 10. Variación de la biomasa $\left(\mu \mathrm{g} . \mathrm{L}^{-1}\right)$ de los 2 taxa de rotíferos dominantes en la laguna Don Tomás, durante el período febrero a diciembre de 2006.

Figure 10. Variation of biomass ( $\mu \mathrm{g} . \mathrm{L}^{-1}$ ) of 2 rotifers dominant taxa in Don Tomás pond, during the period February to December 2006.

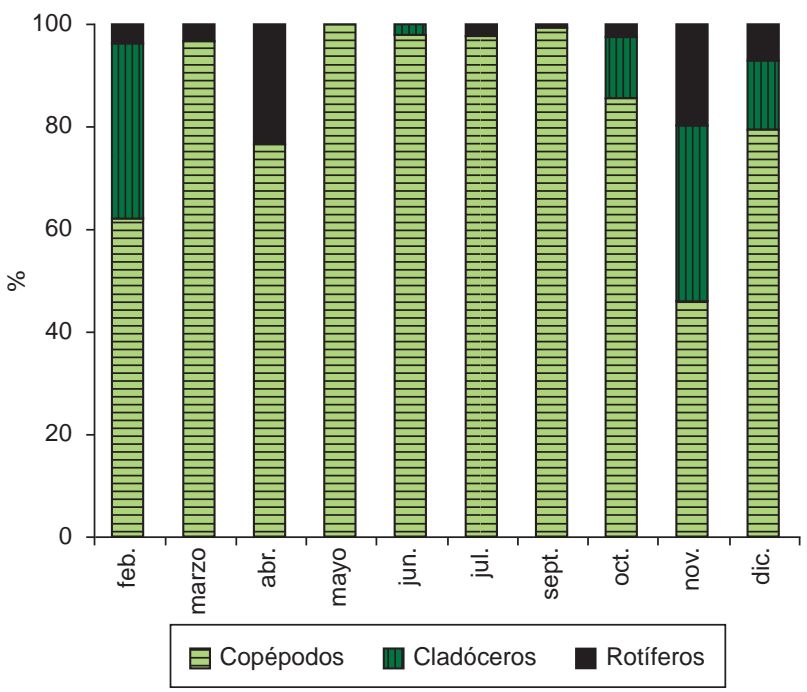

Figura 11. Aporte porcentual de los principales grupos integrantes del zooplancton de la laguna Don Tomás, a la biomasa total, durante el período comprendido entre febrero y diciembre de 2006.

Figure 11. Percentage contribution of major groups of zooplankton components of Don Tomás pond to the total biomass, during the period between February and December 2006.
Al comparar los dos períodos estudiados se evidenció un descenso de la abundancia total de la comunidad zooplanctónica. El test de Kruskal Wallis mostró que la densidad fue significativamente diferente ( $\mathrm{KW}=6,613043 ; \mathrm{p}=0,0101)$, ya que en 2006 fue sólo el $21,8 \%$ de la registrada en $1995 / 96$.

Tomando a los diferentes grupos por separado, el test de Kruskal-Wallis mostró que la abundancia de los rotíferos fue diferente $(\mathrm{KW}=12,21304 ; \mathrm{p}=0,0005)$, ya que en 2006 representó apenas un $3,83 \%$ de lo registrado en 1995/96 (Figura 12). En el caso de los cladóceros, el mismo test $(\mathrm{KW}=5,634783 ; \mathrm{p}=0,0176)$ indicó una situación similar, dado que su densidad fue de $15 \%$ (Figura 12), pero en el caso de los copépodos el test no mostró diferencias significativas ( $\mathrm{KW}=2,300000 ; \mathrm{p}=0,1294$ ) ya que la abundancia medida en 2006 fue el 58\% de la verificada en 1995/96 (Figura 12).

\section{Discusión}

La laguna Don Tomás es un lago somero que se caracteriza por presentar baja salinidad y que, de acuerdo a la clasificación de Hammer (1986), puede caracterizarse como subsalina, categoría que comparte con lagunas de la provincia de condiciones similares, esto es, que reciben aportes pluviales de ciudades, tal el caso de las lagunas Quetré Huitrú, aledaña a General Acha, con una salinidad media de 0,79 g.L L $^{-1}$ (Vignatti \& Echaniz 2007) y la laguna La Arocena cercana a la ciudad de General Pico, con una salinidad de 0,25 g.L L $^{-1}$ (Echaniz \& Vignatti, datos no publicados). Esta situación contrasta con la de la mayor parte de las lagunas de la provincia que no reciben aportes de desagües y tienen salinidades considerablemente más elevadas, la mayor parte de las cuales pueden categorizarse como hipo o mesosalinas (Echaniz et al. 2005, 2006, Vignatti \& Echaniz 2007). Además, durante el estudio realizado en 2006 en Don Tomás este parámetro se mantuvo muy constante, lo que también es diferente a lo que sucede en ambientes alejados de ciudades, en donde se verifican grandes fluctuaciones de salinidad.

La baja salinidad actual de la laguna Don Tomás y su cambio a lo largo del tiempo podría deberse a la influencia ejercida por la

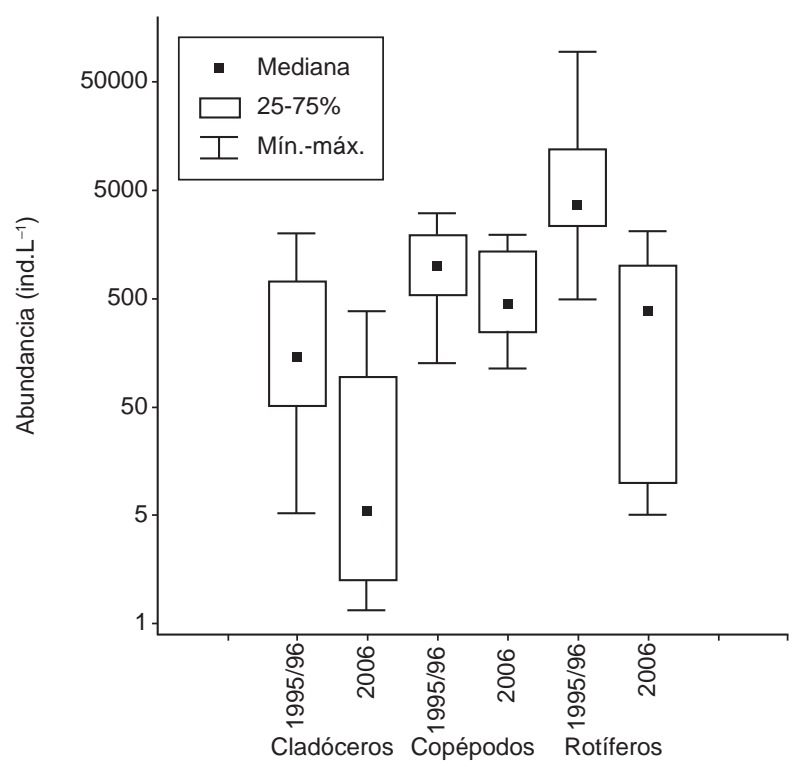

Figura 12. Comparación de la abundancia (ind. $\mathrm{L}^{-1}$ ) de los principales grupos integrantes del zooplancton de la laguna Don Tomás, entre el período 1995/96 y 2006.

Figure 12. Comparison of abundance (ind. $\mathrm{L}^{-1}$ ) of the main zooplankton components in Don Tomás pond, during the period between 1995/96 and 2006. 
ciudad de Santa Rosa en su cuenca de drenaje, ya que el aumento en la extensión del pavimento y el número creciente de construcciones impide la infiltración del agua de las precipitaciones en el suelo, por lo que volúmenes grandes de agua escurren superficialmente o por el sistema de desagües pluviales, ingresando a la laguna con una concentración muy baja de sales disueltas. Esto ha hecho necesaria la construcción de un sistema de derivación de agua hacia un cuenco situado al sur de la ciudad y su puesta en marcha a partir del año 2000 a efectos de evitar inundaciones en zonas habitadas cercanas. Al efecto de lavado dado por el trasvase debe sumarse la continua extracción de agua para el riego de calles mediante camiones.

Teniendo en cuenta el contenido iónico del agua, durante 2006 Don Tomás puede clasificarse como bicarbonatada sódica, mientras que en el período 1995/96 la composición fue diferente, con el predominio de cloruro (Figura 5). En función de la relación calculada entre los cationes mono y bivalentes, puede considerársela de baja dureza (Golterman 1978). Esta situación difirió entre ambos períodos, ya que el valor calculado en 1995/96 fue superior a 8 contra 3,86 en 2006, indicando un cambio en la composición química dado por el marcado descenso de la concentración de sodio.

Tanto por la preponderancia de bicarbonato como por la baja dureza, Don Tomás es similar a las otras lagunas aledañas a ciudades, ya mencionadas, o a algunas situadas en zonas medanosas de la provincia, alimentadas por descargas de la freática (Vignatti \& Echaniz 2007, Echaniz \& Vignatti, datos no publicados).

Don Tomás es un cuerpo de agua que se caracteriza por su elevado estado trófico. Los índices calculados para 2006, teniendo en cuenta la transparencia del agua y las concentraciones de clorofila $a$ y fósforo total, permiten caracterizarla como hipereutrófica (Carlson \& Simpson 1996). El índice calculado para 1995/96, empleando la profundidad del disco de Secchi, arrojó un valor de 80,6, que comparado con el valor actual, basado en el mismo parámetro, único comparable entre los dos períodos, muestra que, si bien la laguna sigue siendo hipereutrófica, ha incrementado su nivel trófico.

Teniendo en cuenta el modelo de los estados alternativos de los lagos someros, la laguna Don Tomás puede ser categorizada como turbia. La escasa transparencia está dada por la alta cantidad de materiales sólidos en suspensión (seston). El test de Kruskal-Wallis (KW = 8,879556; $\mathrm{p}=0$,0029) mostró que este parámetro también registró cambios significativos con respecto al período 95/96, cuando se verificó una transparencia media de $0,24 \mathrm{~m}$ contra los 0,15 medidos durante 2006.

La determinación de la profundidad de la zona fótica, mediante la multiplicación de la profundidad del disco de Secchi $\left(\mathrm{Z}_{\mathrm{SD}}\right)$ por un factor es un parámetro útil, ya que indica hasta donde una laguna puede ser colonizada por macrófitas que contribuyan a crear refugios para el zooplancton con capacidad de aclarar el agua (Quirós 2002). Para el cálculo se empleó el factor 3,3, sugerido por Kalff (2002) para ambientes turbios, y se verificaron diferencias entre los dos períodos en la profundidad a la que puede penetrar la luz, ya que se redujo de 0,79 m en $1995 / 96$ a 0,49 m en 2006.

Dado que el índice obtenido al calcular la relación $\mathrm{Z}_{\text {promedio }} / \mathrm{Z}_{\text {fótico }}$ sirve para diferenciar lagunas claras $(<1)$ de turbias $(>1)$ (Quirós 2002), la comparación de los valores de ambos períodos (1,03 para $1995 / 96$ y 2,35 en 2006) muestra un desmejoramiento en las condiciones de la laguna.

En 2006, la cantidad de sólidos suspendidos de origen inorgánico fue siempre reducida, indicando que la proporción de sedimentos en suspensión es baja. En cambio la fracción sestónica de origen orgánico fue siempre mayor. Dado que se verificó una relación directa entre este componente y la concentración de clorofila a (coeficiente de correlación $=0,8414$ ), mayor que con la biomasa del zooplancton (coeficiente de correlación $=0,3264$ ) puede inferirse que la mayor parte del material particulado sólido suspendido está dado por el fitoplancton.

El estado turbio de Don Tomás se ve favorecido no sólo por las características antrópicas de la laguna y su estrecha relación con la ciudad, que hacen que reciba cantidades altas de nutrientes sino también por el efecto producido por la presencia de pejerreyes, peces planctívoros que predan sobre las especies planctónicas de mayor tamaño y eficiencia de filtración, preferentemente del género Daphnia (Quirós 2002, Grosman \& Sanzano 2003), haciendo que la comunidad zooplanctónica esté integrada sólo por especies de talla reducida y baja eficacia de filtrado.

Esta situación es similar a la registrada en otras lagunas de La Pampa que tienen poblaciones de pejerreyes, como Quetré Huitrú (Vignatti et al. 2007) y Bajo de Giuliani (Echaniz, datos no publicados), que también mostraron una reducida transparencia del agua y comunidades zooplanctónicas caracterizadas por la dominancia de especies de pequeña talla, pero contrasta con las condiciones que presentan otros lagos someros estudiados en La Pampa, los que a pesar de tener similares o superiores concentraciones de nutrientes, presentan una transparencia del agua mucho mayor, que puede ser atribuida a la ausencia de peces planctívoros, lo que permite el desarrollo de especies zooplanctónicas de mayor tamaño y eficiencia de filtración (Scheffer 1998), en particular Daphnia menucoensis Paggi, 1996 y Moina eugeniae Olivier, 1954 (Echaniz et al. 2005, 2006; Vignatti et al. 2007).

La mayor biomasa de la comunidad zooplanctónica fue aportada por los copépodos ciclopoideos, siempre presentes. Durante 2006, la biomasa zooplanctónica mostró una correlación positiva con la temperatura del agua $(0,6106)$, ya que fue mayor en los meses más calidos y menor en los meses más fríos, presentando un pico a fines de primavera - inicios del verano. La correlación calculada entre la biomasa zooplanctónica y la concentración de clorofila $a$ mostró un menor nivel de significación $(0,4029)$, por lo que puede considerarse que, dada la alta concentración de clorofila $a$ que se registró durante todo el período de estudio, el fitoplancton que sirve de alimento al zooplancton nunca fue un factor limitante.

La biomasa media del macrozooplancton de la laguna Don Tomás, si bien está comprendida en el rango establecido por Quirós (2002) para las lagunas turbias de la provincia de Buenos Aires, es superior a la media correspondiente a esa categoría. Esto estaría relacionado con la mayor concentración de nutrientes que posee la laguna Don Tomás, ya que, en el caso de las lagunas bonaerenses el máximo valor de fósforo total registrado fue de $1,25 \mathrm{mg} . \mathrm{L}^{-1}$, mientras que en Don Tomás ese nutriente presentó un valor medio, a lo largo del período de estudio de 9,7 mg.L. $\mathrm{L}^{-1}$. Por otro lado, esta mayor concentración de fósforo permitió la producción de una mayor concentración de clorofila $a$, ya que en la provincia de Buenos Aires el valor medio indicado por el mencionado autor fue de $101 \mathrm{mg} \cdot \mathrm{m}^{-3}$, mientras que en Don Tomás, el valor medio fue de 154,6 mg.m ${ }^{-3}$.

Considerando otros cuerpos de agua de características similares, la biomasa de Don Tomás también fue mayor que la determinada para la laguna bonaerense de San Miguel del Monte (Claps et al. 2004), aunque en ambos casos estuvo dominada por los copépodos ciclopoideos.

La diversidad registrada en Don Tomás fue más baja que la de otras lagunas hiposalinas de La Pampa. El número de especies de esta laguna fue la mitad que el verificado en Quetré Huitrú, donde se registraron 35 especies a lo largo de un ciclo anual (Vignatti et al. 2007) o en El Guanaco (Echaniz \& Vignatti, datos no publicados), donde durante el hidroperíodo que tuvo lugar entre diciembre de 2003 y marzo de 2004 se registraron 34 especies. La riqueza de Don Tomás es más cercana a la que se registra en lagunas pampeanas de mayor salinidad (Echaniz et al. 2005, 2006). 
Durante los períodos 1995/96 y 2006 se registró el mismo número total de especies, 20 en ambos casos, pero se verificaron diferencias en la composición taxonómica. Mientras en el primer ciclo la comunidad zooplanctónica estuvo dominada por los microcrustáceos, ya que se contaron 12 especies, contra 8 de rotíferos, en 2006 la situación fue inversa, dado que se registraron 8 taxa de crustáceos contra 12 de rotíferos.

Si bien algunas especies, como Bosmina huaronensis, Metacyclops mendocinus, Microcyclops anceps y Keratella sp. fueron de presencia constante durante ambos períodos, en 1995/96 se verificó la presencia durante 5 meses de dos especies del género Daphnia (D. spinulata y D. obtusa), completamente ausentes durante 2006 (Tabla 1).

Las diferencias en la composición taxonómica y en la abundancia del zooplancton de los dos períodos en que se estudió la laguna Don Tomás, dadas, tanto por la desaparición de especies de crustáceos de talla grande y mayor eficiencia de filtración como por la disminución significativa de la abundancia, además de tener influencia en la disminución de la transparencia del agua por menor pastoreo, son indicativas de un empobrecimiento de la comunidad, pudiendo estar producido por la predación ejercida por los peces (Claps et al. 2004, Mancini \& Grosman 2004, Grosman \& Sanzano 2003) a lo largo de la década que medió entre ambos estudios.

\section{Agradecimientos}

A la Facultad de Ciencias Exactas y Naturales de la Universidad Nacional de La Pampa por el financiamiento brindado. Al revisor anónimo que colaboró en el mejoramiento del manuscrito.

\section{Referencias Bibliográficas}

ALVAREZ, S., BIASOTTI, A., BERNARDOS, J. \& BAZÁN, G. 2006. Ficoflora de la laguna Don Tomás (La Pampa, Argentina). Biol. Acuát. 22:1-6.

APHA. 1999. Standard Methods for the Examination of Water and Wastewater, 20. American Public Health Association (APHA), American Water Works Association (AWWA) and Water Pollution Control Federation (WPCF), Washington, DC.

ARAR, E.J. 1997. In Vitro Determination of Chlorophylls a, b, c + $\mathrm{c}$ and Pheopigments in Marine and Freshwater Algae by Visible Spectrophotometry. Method 446.0. U.S. Environmental Protection Agency.

BAYLY, I.A.E. 1992a. Fusion of the genera Boeckella and Pseudoboeckella (Copepoda) and revision of their species from South America and subAntarctic islands. Rev. Chil. Hist. Nat. 65:17-63.

BAYLY, I.A.E. 1992b. The non-marine Centropagidae (Copepoda: Calanoida) of the world. In Guides to the Identification of the Microinvertebrates of the Continental Waters of the World (H. J. Dumont, ed.). SPB Academic Publishers, The Hague.

BOVERI, M.B. \& QUIRÓS, R. 2002. Trophic interactions in pampean shallow lakes: evaluation of silverside predatory effects in mesocosm experiments. Verh. Internat. Verein. Limnol. 28:1-5.

CARLSON, R.E. \& SIMPSON, J. 1996. A coordinator's Guide to Volunteer Lake Monitoring Methods. North American Lake Management Society, Madison, Wisconsin.

CLAPS, M.C., GABELLONE, N.A. \& BENÍTEZ, H.H. 2004. Zooplankton biomass in an eutrophic shallow lake (Buenos Aires, Argentina): spatio - temporal variations. Ann. Limnol.-Int. J. Lim. 40(3):201-210.

CULVER, D.A., BOUCHERLE, M.M., BEAN, D.J. \& FLETCHER, J.W. 1985. Biomass of freshwater crustacean zooplankton from length- weight regressions. Can. J. Fish. Aquat. Sci. 42(8):1380-1390.

DANGAVS, N. 1995. Morfometría de cuerpos lénticos. In Ecosistemas de aguas contientales. Metodologías para su estudio (E. Lopretto \& G. Tell, eds). Ediciones Sur, La Plata.
DUMONT, H.J., VAN DER VELDE, I. \& DUMONT, S. 1975. The dry weigth estimate of biomass in a selection of Cladocera, Copepoda and Rotifera from the plankton, periphyton and benthos of continental waters. Oecologia (Berlin) 19:75-97.

ECHANIZ, S.A. \& VIGNATTI, A.M. 1996. Cladóceros limnéticos de la provincia de La Pampa (Argentina). Rev. Fac. Agronomía. UNLPam. 9(1):65-80.

ECHANIZ, S.A. \& VIGNATTI, A.M. 2001. Composición y variación anual de la taxocenosis de cladóceros (Crustacea: Anomopoda y Ctenopoda) planctónicos y química del agua de la laguna Don Tomás (La Pampa, Argentina). Rev. Fac. Agronomía 12(2):23-35.

ECHANIZ, S.A. \& VIGNATTI, A.M. 2002. Variación anual de la taxocenosis de cladóceros planctónicos (Crustacea: Branchiopoda) de una laguna de elevada salinidad (La Pampa, Argentina). Neotrópica 48:11-17.

ECHANIZ, S., VIGNATTI, A., JOSÉ DE PAGGI, S. \& PAGGI, J. 2005. Riqueza y composición del zooplancton de lagunas saladas de la región pampeana argentina. Revista FABICIB 9:25-39.

ECHANIZ, S., VIGNATTI, A., JOSÉ DE PAGGI, S., PAGGI, J. \& PILATI, A. 2006. Zooplankton seasonal abundance of south american saline shallow lakes. Internat. Rev. Hydrobiol. 91(1):86-100.

EPA (ENVIRONMENTAL PROTECTION AGENCY).1993. ESS Method 340.2: Total Suspended Solids, Mass Balance (Dried at 103 - 105 C) Volatile Suspended Solids (Ignited at 550 C). Environmental Sciences Section, Wisconsin State Lab of Higiene, Madison, Wisconsin.

GOLTERMAN, H.L., CLYMO, R.S. \& OHNSTEAD, M.A.M. 1978. Methods for physical and chemical analysis of freshwaters. 2nd ed. Blackwell Sc. Publ, Oxford-Edimburgh-London.

GÓMEZ, S., FLEEGER, J.W., ROCHA-OLIVARES, A. \& FOLTZ, D. 2004. Four new species of Cletocamptus Schmankewitsch, 1875, closely related to Cletocamptus deitersi (Richard, 1897) (Copepoda: Harpacticoida). Journ. Nat. History 38(21):2669 - 2732.

GOULDEN, C.E. 1968. The systematics and evolution of the Moinidae. Trans. Amer. Phil. Soc. 58(6):1-101.

GROSMAN, F. \& SANZANO, P. 2003. ¿El pejerrey puede causar cambios estructurales en un ecosistema? Biol. Acuát. 20:37-44.

HAMMER, U.T. 1986. Saline Lake Ecosystems of the World. Monographiae Biologicae 59. Dr. W. Junk Publishers, Dordrecht.

JEPPESEN, E. 1998. The ecology of shallow lakes- trophic interactions in the pelagial. Dissertation, National Environmental Research Institute, Silkeborg, Denmark. NERI Technical Report 247.

JEPPESEN, E., KRISTENSEN, P., JENSEN, J.P., SØNDERGAARD, M., MORTENSEN, E. \& LAURIDSEN, T. 1991. Recovery resilience following a reduction in external phosphorus loading of shallow, eutrophic Danish lakes: duration, regulation factors and methods for overcoming resilience. Mem. Ist. Ital. di Idrobiol. 48:127-148.

JEPPESEN, E.J., JENSEN P., SøNDERGAARD, M., LAURIDSEN, T. \& LANDKILDEHUS, F. 2000. Trophic structure, species richness and biodiversity in Danish lakes: changes along a phosphorus gradient. Freshwat. Biol. 45(2):201-218.

JOSÉ de PAGGI, S. 1976. Distribución espacial y temporal del zooplancton de un cuerpo de agua eutrófico (Lago del Parque Gral. Belgrano, Santa Fe). Physis (B) 35(91):171-183.

JOSÉ de PAGGI, S. 1980. Campaña limnológica “Keratella I” en el río Paraná medio: Zooplancton de ambientes lóticos. Ecología 4:69-75.

JOSÉ DE PAGGI, S. 1983. Estudio sinóptico del zooplanton de los principales cauces y tributarios del valle aluvial del río Paraná: tramo Goya- Diamante (I Parte). Rev. Asoc. Cien. Nat. Litoral 14(2):163-178.

JOSÉ DE PAGGI, S. 1984. Estudios limnológicos en una sección transversal del tramo medio del río Paraná. X: Distribución estacional del zooplancton. Rev. Asoc. Cien. Nat. del Litoral 15(2):135-155.

JOSÉ DE PAGGI, S. \& PAGGI, J.C. 1995. Determinación de la abundancia y biomasa zooplanctónica. En: Lopretto, E. y G. Tell (eds.). Ecosistemas de aguas continentales. Metodologías para su estudio. Tomo 1. Ed. Sur, La Plata, Argentina, p. 315- 323. 
KALFF, J. 2002. Limnology: inland water ecosystems. Prentice -Hall. Inc, New Jersey.

KOBAYASHI, T. 1997. Associations between environmental variables and zooplankton body masses in a regulated Australian river. Mar. Freshwater Res. 48:523-529.

KOROVCHINSKY, N.M. 1992. Sididae y Holopedidae (Crustacea: Daphniiformes). Guides to Identification of the Microinvertebrates of the Continental Waters of the World. III. SPB Academic Publishing, The Hage.

KOSTE, W. 1978. Rotatoria. Die Radertiere Mitteleuropas. Gebruder Borntraege, Berlin

MCCAULEY, E. 1984. The estimation of the abundance of biomass of zooplankton in samples. In Downing, J.A. \& F. Rigler (eds.). A manual on methods for the assessment of secondary productivity in freshwaters. 2. ed. Blackwell, Oxford, p. 228-265.

MANCINI, M. \& GROSMAN, F. 2004. Estructura y funcionamiento de la pesquería recreacional del pejerrey (Odonthestes bonariensis) en la laguna de Suco (Córdoba) Argentina. Rev. AquaTIC (20):20-31.

OLIVIER, S.R. 1955. Contribution to the limnological knowledge of the Salada Grande lagoon. In: Proceedings International Association of Limnology, 12:302-308.

OLIVIER, S.R. 1961. Estudios limnológicos de la laguna Vitel (Pdo. de Chascomús-Buenos Aires-Argentina). Agro 6:1-128.

PAGGI, J.C. 1980. Campaña limnológica "Keratella I" en el río Paraná medio (Argentina): Zooplancton de ambientes leníticos. Ecología 4:77-88.

PAGGI, J.C. 1995. Cladocera. In Ecosistemas de aguas continentales. Metodologías para su estúdio (E. LOPRETTO \& Y. G. TELL, eds.). Ediciones Sur, La Plata.

PAGGI, J.C. 1998. Cladocera (Anomopoda y Ctenopoda). En: (S. Coscarón \& J.J. Morrone, eds) Biodiversidad de Artrópodos Argentinos. Ediciones Sur, La Plata.

PILATI, A. 1997. Copépodos calanoideos de la provincia de La Pampa. Rev. Fac. Agron. UNLPam., 9(2):57-67.

PILATI, A. 1999. Copépodos cyclopoideos de la provincia de La Pampa. Rev. Fac. Agron. UNLPam., 10(1):29-44.

PILATI, A. \& MARTÍNEZ, J.J. 2003. Relación longitud- peso de siete especies de Boeckella (Copepoda: Calanoida) de la República Argentina. Neotrópica 49:55-61.

QUIRÓS, R. 2000. La eutrofización de las aguas continentales de la Argentina. I Reunión de la Red temática sobre Eutrofización de Lagos y Embalses. Mar del Plata, Argentina.

QUIRÓS, R., RENNELLA, A., BOVERI, M., ROSSO J. \& SOSNOVSKY, A. 2002a. Factores que afectan la estructura y el funcionamiento de las lagunas pampeanas. Ecol. Aust. 12:175-185.

QUIRÓS, R., ROSSO, J.J., RENNELLA, A., SOSNOVSKY, A. \& BOVERI, M. 2002b. Sobre el estado trófico de las lagunas pampeanas (Argentina).
In: Reunión Internacional de Eutrofización de Lagos y Embalses. Fac. de Ciencias, Universidad de la República, Montevideo, Uruguay.

QUIRÓS, R., ROSSO, J.J., RENNELLA, A., SOSNOVSKY, A. \& BOVERI, M. 2002c. Análisis del estado trófico de las lagunas pampeanas (Argentina). Interciencia 27(11):584-591.

REID, J.W. 1985. Chave de identificação e lista de referências bibliográficas para as espécies continentais sulamericanas de vida livre da ordem Cyclopoida (Crustacea, Copepoda). Bolm. Zool., USP 9:17-143.

RINGUELET, R.A. 1958. Los Crustáceos Copépodos de las aguas continentales de la República Argentina. Sinopsis sistemática. Contrib. Cient. Fac. Cs. Ex. Fis. y Nat. UBA, Zool., 1:35-126.

RINGUELET, R.A. 1962. Ecología acuática continental. Manuales de la Editorial Universitaria de Buenos Aires (EUDEBA), Buenos Aires.

RINGUELET, R.A. 1972. Ecología y Biocenología del habitat lagunar o lago de tercer orden de la región neotrópica templada (Pampasia Sudoriental de la Argentina). Physis 31(82):55-76.

RINGUELET, R.A., MORENO, I. \& FELDMAN, E. 1967. El zooplancton de las lagunas de la pampa deprimida y otras aguas superficiales de la llanura bonaerense (Argentina). Physis 27(74):187-200.

ROSEN, R.A. 1981. Length - dry weight relationships of some freshwaters zooplankton. J. Freshw. Ecol. 1:225-229.

RUTTNER-KOLISKO, A. 1974. Plankton rotifers; Biology and taxonomy. Die Binnengewässer 26 (1):1-146.

RUTTNER-KOLISKO, A. 1977. Suggestions for biomass calculation of plankton rotifers. Arch. Hydrobiol. Beih (8):71-76.

SCHEFFER, M. 1998. Ecology of Shallow Lakes. Chapman \& Hall, London.

SCHEFFER, M., HOSPER, S.H., MEIJER, M.L., MOSS B. \& JEPPESEN, E. 1993. Alternative equilibria in shallow lakes. Trends Ecol. Evol. 8:275-279.

SEGERS, H. 1995. Rotifera 2. The Lecanidae (Monogononta). In Guides to the Identification of the Microinvertebrates of the Continental Waters of the World 6. (H.J. Dumont \& T. Nogrady, eds). SPB Academic Publishing, The Hague.

SMIRNOV, N.N. 1971. Chydoridae fauni mira. Fauna SSSR, Crustacea $1(2): 1-531$.

VignATTI, A.M. \& ECHANIZ, S. 1999. Presencia de Daphnia (Ctenodaphnia) menucoensis Paggi, 1996 en la provincia de La Pampa (Argentina). Rev. Fac. Agronomía, UNLPam 10(1):21-27.

VIGNATTI, A., ECHANIZ, S. \& MARTÍN, M.C. 2007. El zooplancton de tres lagos someros de diferente salinidad y estado trófico en la región semiárida pampeana (Argentina). Gayana 71(1):34-48.

ZAR, J.H. 1996. Bioestatistical analysis. 3. ed. Prentice Hall, New Jersey.

Recebido em 23/11/07

Versão reformulada recebida em 15/10/08

Publicado em 28/10/08 